\title{
Use of amnion chorion and umbilical cord grafts in conjunction with penile implant procedures
}

\author{
Andrew C. Kramer \\ Division of Urology, Department of Surgery, University of Maryland School of Medicine, Baltimore, MD, USA \\ Correspondence to: Dr. Andrew Kramer, MD, MBA. Division of Urology, Department of Surgery, University of Maryland School of Medicine, \\ Baltimore, MD, USA. Email: AKramer@som.umaryland.edu.
}

\begin{abstract}
Biologics have increased in popularity lately as a novel and exciting new therapy for men with a spectrum of sexual dysfunction ailments_ranging from erectile dysfunction, Peyronie's disease, and ejaculatory disorders. In this series, sequential patients were analyzed who received biologic materials at the time of their penile implant surgery, with various metrics assessed. The information gathered includes recovery time, need for postoperative analgesics, and satisfaction with the final outcome. In this study, early metrics appear to point to improved outcomes in select criteria. Further study will need to address whether treatment with biologics has broader appeal, but in the penile implant patient, the surgery seems to be augmented with the placement of biologic material into the surgical wound.
\end{abstract}

Keywords: Biologics; erectile dysfunction; sexual medicine; stem cells

Submitted Jul 31, 2017. Accepted for publication Nov 07, 2017.

doi: $10.21037 /$ tau.2017.11.05

View this article at: http://dx.doi.org/10.21037/tau.2017.11.05

\section{Introduction}

"Biologics" have been touted in recent years as a promising new therapy, a novel pathway, and perhaps the most promising development in the field of male sexual dysfunction since the invention of Viagra. There are important distinctions in the nomenclature of biologics. Amnion/Chorion/Umbilical Cord Allografts are thought to have growth factors from Stem Cells but no living Stem Cells. "Stem Cells" are undifferentiated cells that can still differentiate into specialized cells, may promote vasculogenesis and promote wound healing (1). Stem cells are derived from bone marrow, or may be adipose derived or placental derived (2). In our study, biologics were used, which may be derived from amniotic fluid or membrane, or umbilical cord (3). The promise of implanting biologics is still potential improvement in wound healing and improved infection control, something enticing for the penile implant surgeon. In this case, 20 subsequent patients received penile implants, and in the wound, was placed a Cygnus Max or a Cygnus Matrix product. The Cygnus Max consists of umbilical cord derived tissue, while the Cygnus Matrix is derived from placenta.

\section{Methods}

This study was structured as a retrospective analysis, in a single high volume penile implant practice. The goal was to change only one variable, the use and insertion of the biologic allograft. This sheet containing the biologic material had never been used to this author's knowledge in conjunction with penile prosthesis implantation. The materials, Cygnus Max and Cygnus Matrix, were approved and ordered by the tissue bank at this major tertiary academic medical center. Informed consent was obtained from all patients. There was an extremely high degree of confidence with the safety and sterility of the materials, since the product was irradiated and sterilized appropriately, but it was unclear if there would be any benefit at all, and if so, where should the material be placed so that a benefit could be derived.

Therefore in 20 consecutive procedures, where patients were "virgin" penile implant patients, meaning first timenot revisions, the product was used. In the first 5 cases, 
the sheet containing the biologic cells was inserted in the scrotum at the very end of the procedure, just prior to closing dartos muscle. In the next and final 15 patients, the material was cut into 2 pieces, and each was placed over the corporotomy where the cylinder was placed, and was sutured in with the corporotomy suture. This was felt to secure its location, and also derive maximal benefit. Since the thought was to maximize recruitment and recovery of vascular structures, promote wound healing, and possibly stimulate the immune system to minimize infection, the corporotomy seemed like the most high yield location.

Questionnaires were given to each patient, which was exactly the one given to all patients in this practice- since data is collected and studied for nearly all patients in this practice. Data has been collected on penile implant patients in this practice for over 10 years, owing to the fact that there is a high volume of a very specific surgical procedure being done, and historically this procedure had been done in private practice settings where little data had been collected.

Questions focused on:

* Amount of narcotic medication used in the postoperative period of 28 days;

* Time to "activation", or ability to inflate and deflate the penile implant without discomfort.

The presence or absence of complications, including infection, hematoma, or wound dehiscence.

All patients were seen at the 2-week point, and the 4-week point, per standard practice protocol. The study was not blinded, so the patients who received the sheets of biologic material were told so prior to be enrolled.

\section{Results}

During the course of this retrospective study, there were no reported cases of complications. There were no infections, and no wound or tissue reactions. The product appeared to be safe, at least within the first 28 days.

\section{Hematomas}

Of the 20 patients in the "study group", in whom the biologic material was introduced, 3 patients self-reported small hematomas, that were confirmed at the 2-week office. All 3 then recovered and the hematoma had reabsorbed by the 4 week visit. In these cases, the hematomas were subjectively small in size, approximately $3 \times 3 \mathrm{~cm}$ in size in all cases. This is in line with historical practice outcomes, where the hematoma rate throughout the past 10 years or so has been $22 \%$. Therefore the study group had a $15 \%$ hematoma rate, in an extremely small sample size. Nevertheless, it is important to note items that were not seen, such as this. Hematoma formation was thought to be less relevant than other metrics, studied below.

\section{Narcotic usage}

The study group had lower narcotic requirements than their cohorts in the control group. The average narcotic requirement of the control group has trended down over the past 10 years significantly, but the past year demonstrates use of about $105 \mathrm{mg}$ tablets, or $50 \mathrm{mg}$ total. The 20 patients studied here who received sheets of the biologic material incorporated into the penile implant procedure used on average only 4 pills, or $20 \mathrm{mg}$.

\section{Time to activation}

The most clinically significant metric studied, and indeed the factor that demonstrated the most difference, was how many days after surgery until a patient could be comfortable squeezing the pump enough to fully inflate and deflate their device. Utilizing the most recent 2 years of data for the control group, the time to activation was $22.3 \pm 1.4$ days. This is studied by giving detailed, hands on instructions at the 2-week appointment, including use of a keychain, one on one teaching, and daily attempts at cycling for patients who are not quite ready to manipulate the pump at that 2 -week checkup. At the 28 day follow up, the date is collected from patients on the time period that they can realistically consider themselves healed and pain-free enough to inflate and deflate in a meaningful manner. The time to activation for the 20 patients in the experimental group was $16.1 \pm 0.8$ days.

\section{Discussion}

Biologics as a whole, including stem cell treatments, have gained a tremendous amount of traction and appeal to patients in whom traditional options are acceptable, but outside the box therapies do not seem unreasonable $(4,5)$. A shortcoming of this study is clearly the small sample size, leading to almost "anecdotal" results. A larger, double blind, placebo controlled, prospective and multi-institutional study would solve whether or not this treatment does in fact reduce the pain of the operation and improve recovery (6). However, it appears at least that there are minimal to no 
untoward complications, and outcomes do not seem worse than their counterparts in the control group.

The appeal in this therapy was more significant than anticipated. Patients, upon hearing that the therapy was available and could be given at no extra cost, were inquiring by the hundreds, and flying in for surgery in hopes of the possibility of receiving biologic therapies with their penile implant procedure. Since most procedures are videotaped and disseminated in this practice, it became known that this therapy was being offered, and the interest grew rather quickly- speaking to the appeal of this new therapy, even though results are largely unproven.

The advantage of earlier activation is potentially great $(7,8)$. Early cycling means that patients can use the tissue expander qualities of the device at a point when tissues of the penis are still plastic, thus offering the potential for more length and girth (9). Further clinical trials are needed to verify if cycling penile implants, and indeed early cycling, leads to increased penile length and girth. Clearly, the topic has widespread appeal in surgery, specifically penile implant procedures, but larger and more technical studies will be required to achieve better data and more answers.

\section{Acknowledgements}

None.

\section{Footnote}

Conflicts of Interest: The author has no conflicts of interest to declare.

Informed Consent: Written informed consent was obtained from the patient for publication of this case report and any accompanying images.

Cite this article as: Kramer AC. Use of amnion chorion and umbilical cord grafts in conjunction with penile implant procedures. Transl Androl Urol 2017;6(Suppl 5):S900-S902. doi: $10.21037 /$ tau.2017.11.05

\section{References}

1. Carrier S, Nagaraju P, Morgan DM, et al. Age decreases nitric oxide synthase-containing nerve fibers in the rat penis. J Urol 1997;157:1088-92.

2. Lewis RW, Fugl-Meyer KS, Corona G, et al. Definitions/ epidemiology/risk factors for sexual dysfunction. J Sex Med 2010;7:1598-607.

3. Castiglione F, Hedlund P, Van der Aa F, et al. Intratunical injection of human adipose tissue-derived stem cells prevents fibrosis and is associated with improved erectile function in a rat model of Peyronie's disease. Eur Urol 2013;63:551-60.

4. Mangir N, Akbal C, Tarcan T, et al. Mesenchymal stem cell therapy in treatment of erectile dysfunction: autologous or allogeneic cell sources? Int J Urol 2014;21:1280-5.

5. Levy JA, Marchand M, Iorio L, et al. Effects of Stem Cell Treatment in Human Patients With Peyronie Disease. J Am Osteopath Assoc 2015;115:e8-13.

6. Levy JA, Marchand M, Iorio L, et al. Determining the Feasibility of Managing Erectile Dysfunction in Humans With Placental-Derived Stem Cells. J Am Osteopath Assoc 2016;116:e1-5.

7. Sopko N, Matsui H, Kates M, et al. Stromal Derived Factor-1 treatment augments nerve regeneration via CXCR4 activation of neurotrophic factors in the major pelvic ganglion in a dose-dependent fashion. J Urol 2016;195:e1139-40.

8. Bennett NT, Schultz GS. Growth factors and wound healing: Biochemical properties of growth factors and their receptors. Am J Surg 1993;165:728-37.

9. Efficacy proven in 2 Large Multicenter Studies with Xiaflex. 2016 (July). Available online: http://peyroniesdisease.xiaflex.com/hcp/clinical-data/ 